\title{
Highly concentrated emulsion with a cubic liquid crystal as the external phase: characterization and obtaining of meso/macroporous material
}

\author{
Santamaría. E, Cortés. M, Maestro.A, Porras.M, Gutiérrez, J.M and González. C* \\ Department of Chemical Engineering ,Faculty of Chemistry, University of Barcelona, Barcelona 08028, \\ Martí i Franqués 1-11, Spai,E-mail: carme.gonzalez@ub.edu
}

\begin{abstract}
High internal phase ratio emulsions (HIPRE) or highly concentrated emulsions are characterized by their large internal phase volume.The nature and concentration of surfactant affects several features of the final emulsion, such as stability, droplet size and structure of the external phase. Therefore, an ordered external mesophase (structure of liquid crystal) can be obtained.. The present work studies the influence of composition and preparation variables on the final properties of HIPRE with an external phase formed by a bicontinuous cubic liquid crystal. The studied outputs variables were droplet size, stability and rheological parameters as yield stress, viscosity at a fixed shear rate and the plateau value of the storage modulus. O/W HIPREs were prepared with a decane/brij 35/water system. Once the emulsions were characterized the meso/macroporous material was obtained. In order to prepare the materials, the emulsions were formed by incorporating the catalyst of the reaction $(\mathrm{HCl})$ in the continuous phase. When the emulsions were formed, tetraethylorthosilicate (TEOS) was added as a silica source In order to optimize the production process a study of reutilization and recovery of some of the raw materials (i.e: the surfactant and the EtOH used) was done.
\end{abstract}

Keywords: Mesoporous, macroporous, cubic liquid crystal, emulsion

\section{Introduction}

Highly concentrated emulsions are characterized by a volume in disperse phase higher than 0.74 . This volume corresponds to the maximum packing of uniform and spherical drops. When more disperse phase is added these drops stop being spherical and become polyhedral, separated only by a thin continuous phase ${ }^{1}$.

On the other hand, surfactants and amphiphilic copolymers are well known stabilizing agents in emulsions but they can also give a structure to the continuous phase of the emulsions, such as bicontinuous cubic ones. Moreover, surfactants can be used as templates to synthesize materials with different porosities ${ }^{2-4}$.

Many applications for the mesoporous materials have already been described in literature, such as catalysis supports, adsorption, encapsulation of proteins or biomolecules. This types of applications could require a combination of mesoporous pore sizes, as the normally described for the mesostructured materials like the MCM- $4^{5-6}$ and macroporous pore sizes that improve the diffusion of the reactants and products (favoring the mass transfer while reducing transport limitations). The structural features of these meso-macroporous materials promise uses as potential supports and catalysts in heterogeneous catalysis

* Corresponding author 
for bulkier molecules where diffusion of reactant molecules could be facilitated. A controlled multiporosity network with a high surface area, the wide variety of oxide compositions, and the ability for homogeneous or selective doping of active sites, are in high demand for improved catalytic activity. Currently the applications in reaction processes are emerging.

Chmelka and co-workers prepared meso-macroporous aluminosilicas $(\mathrm{Si} / \mathrm{Al}=72)$ of centimetre sized, mechanically stable monoliths with interconnected macropores whose walls are comprised of ordered mesopores by combining oil-in-water $(\mathrm{O} / \mathrm{W})$ emulsion and block-copolymer templating ${ }^{7}$, and investigated their Friedel-Crafts alkylation properties of single-ring aromatic compounds, including toluene, ethyl-benzene, cumene, and styrene, with benzyl alcohol.

This combination of pore sizes may be obtained from highly concentrated emulsions, with the drops in the disperse phase acting as templates of macropores and structures given by surfactant molecules acting as templates for mesoporous. According to IUPAC ${ }^{8}$ the materials are considered as mesoporous when the pore sizes are between $2-50 \mathrm{~nm}$ and are considered macroporous material when theur pore sizes are bigger than $50 \mathrm{~nm}$.

In order to obtain meso/macroporous materials it is important to study formation of the emulsions and their characterization. Thus this work has been dedicated to the study of the emulsions and to the study of the synthesis of the material using emulsions as templates.

\section{Experimental}

\section{Materials}

Polyoxyethylene dodecyl ether containing 23 oxyethylene units per molecule (designated $\mathrm{C}_{12} \mathrm{EO}_{23}$ ) was supplied by Sigma Aldrich. Moreover n-decane was used as a emulsion dispersed phase and purchased from Quimidroga. Also TEOS (Tetraethylorthosilicate) was used as a silica source and was supplied by Sigma Aldrich. Finally ethanol (96\%v/v) and $\mathrm{HCl}(37 \%)$ were purchased from Panreac. Deionized water was used in all samples. All Chemicals were used without further purification

\section{Methods}

\section{Preparation of the emulsion}

In order to characterize the emulsions a central composite experimental design is done. The analysis consists in a surface design that is applied to the modelization and optimization stages of a process. The total number of experiments to be performed is the sum of a complete factorial design, $2^{\mathrm{k}}$, a central point that is replicated a number of times and an axial or star design that allows estimating the curvature ${ }^{9 .}$ In figure 1 experimental dominion for a central composite design for three variables is shown.

The variables to be studied are the agitation speed (N) [rpm], the addition speed (q) [mL/min] and the disperse phase percentage.

The emulsion was prepared in a jacketed vessel at $70^{\circ} \mathrm{C}$ in order to ensure that the surfactant was melted down and mixed properly with the water, so the continuous phase of the uniform emulsion could be obtained while adding the decane.

\section{Determination of the Phase Behaviour}

In order to establish the phase diagram, the needed amounts of each component were weighted in dry and clean tubes (diameter $=14 \mathrm{~mm}$.). Samples at different concentrations heated above $70^{\circ} \mathrm{C}$. The samples were by means of a vortex. Once homogeneous, the samples were maintained in a thermostatted bath of water at $25^{\circ} \mathrm{C}$ during several weeks until the equilibrium was reached. This process was repeated at different temperatures in order to obtain the phase diagram with the temperatures (Figure 2). Visually it 
was observed an area where the mixture presented a high viscosity, the sample did not flow when the tube was shaken and where the mixture was transparent. It did not present birefringency when it was observed with cross-polarizers.

Moreover the SAXS analysis was done to confirm the liquid crystal phases.

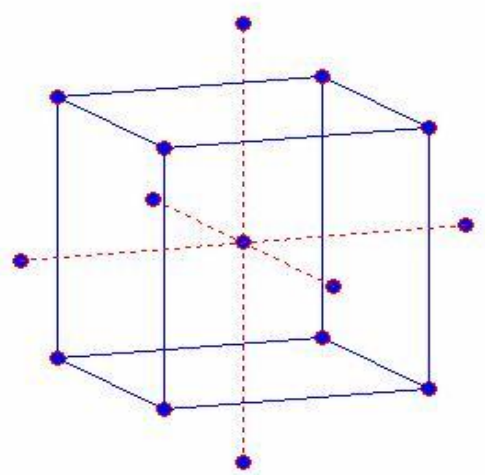

Figure 1: Experimental dominion for a central design composed by three variables

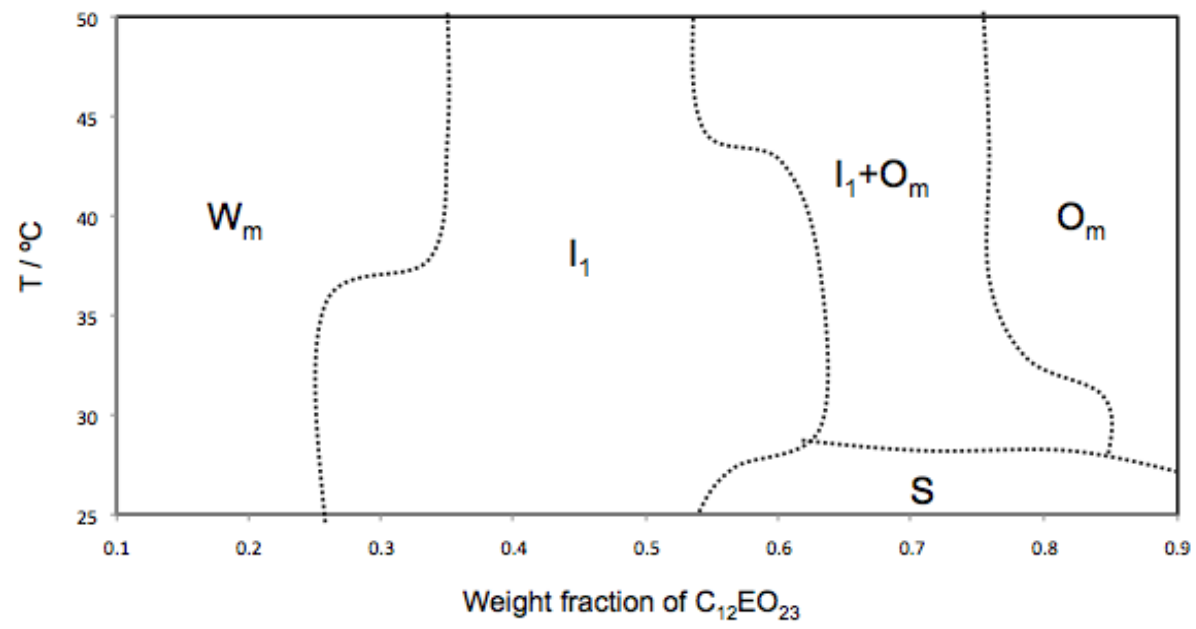

Figure 2: Phase behaviour of the $C_{12} E O_{23}$ /water system. $I_{1}$ indicates a micellar cubic phase, $W_{m}$ a micellar solution, $O_{m}$ a inverse micellar solution, $S$ a solid-present region.

\section{SAXS Measurements}

Small-angle X-ray diffraction scattering (SAXS) measurements were used to confirm the liquid crystal phase. Measurements were performed in a Hecus X-ray Systems GMBH Graz, equipped with a Siemens Kristalloflex 760 (K-760) generator. The temperature of the samples were controlled by a Peltier Anton Paar $\left(25^{\circ} \mathrm{C}-300^{\circ} \mathrm{C}\right)$ controller. Radiation wavelength $1.54 \mathrm{~nm}$.

In the bicontinuous cubic phases, the lattice parameters (a) was calculated with the most intense reflection: 


$$
\mathrm{a}=\left(\mathrm{h}^{2}+\mathrm{k}^{2}+\mathrm{l}^{2}\right)^{1 / 2} \mathrm{~d}
$$

Where h, k y 1 are Miller indexs. The lattice parameter (a) was also equal to the slope in the indexing of the peaks $\left(\mathrm{d}^{-1}\right.$ vs $\left(\mathrm{h}^{2}+\mathrm{k}^{2}+\mathrm{l}^{2}\right)^{1 / 2}$ plot $)$.

\section{Rheological measurements}

Rheological measurements were carried out in a Rheostress 300 rheometer (ThermoHaake) using cone and plate geometry $\left(35 \mathrm{~mm}\right.$ diameter, serrated, $1^{\circ}$, and $1 \mathrm{~mm}$ gap). Temperature was set and kept constant al $25^{\circ} \mathrm{C}$. A frequency sweep test $(0.1-100 \mathrm{rad} / \mathrm{s})$ was performed at a constant strain in the linear viscoelastic zone of the samples, which was determined previously in a strain sweep test. The elastic modulus $\left(G^{\prime}\right)$ and the viscous modulus $\left(G^{\prime \prime}\right)$ of samples were determined. Yield stress was determined $\left(\tau_{0}\right)$ with a stress sweep test.

\section{Results and Discussion}

\section{Phase behaviour of the $\mathrm{C}_{12} \mathrm{EO}_{23}$ /Water System.}

A wide bicontinuous cubic liquid crystal phase region $\left(\mathrm{I}_{1}\right)$ is found in the $\mathrm{C}_{12} \mathrm{EO}_{23} /$ Water system. This behavior is typical of very hydrophilic surfactants and amphphilic polymers ${ }^{[10-11]}$. The liquid crystal zone starts for a Brij 35: water of 0.3:0.7 and it reaches until a 0.65: 0.35. Five very intense peaks were observed by SAXS. The peaks indicate a Pn3m bicontinuous cubic liquid crystal with a lattice parameter of 12.4nm (Figure 3).

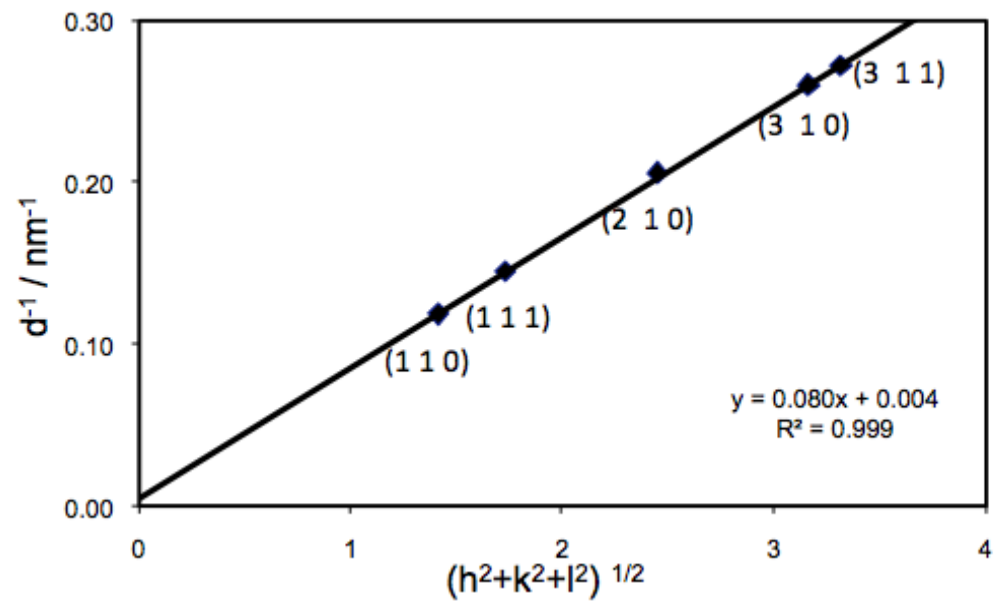

Figure 3: Indexing of SAXS peaks of sample (Brij 35: Water 0.3:0.7). At $25^{\circ} \mathrm{C}$ a Pn3m structure is observed.

\section{Formation and stability of the emulsions}

When the limits of the emulsion formation were studied it was established that it was only possible to obtain emulsion in the lowest surfactant/water relation in which the cubic liquid crystal is present. With a higher relation the resulting mix has a too high viscosity (due to the fact that as the more surfactant present the higher is the number of the spheres forming a more compact structure) so it is difficult to shake it properly in the tank, and when adding the decane it is not dispersed properly. Probably in the 
future it will be possible to make essays with another kind of agitator that allows the dragging of the continuous phase.

It exists a narrow relation between the addition speed and the agitation speed when forming an emulsion. If the adding speed is high and the agitation speed was low it was impossible to form the emulsion because the disperse phase was not suitably incorporated and it remained in the surface. If the adding speed was low and the agitation speed was high then (and due to the viscosity of the aquous phase) the emulsion was not formed because it adhered to the walls of the tank.

To form the emulsion the disperse phase must be added quickly while the cubic liquid crystal is still forming, so it can penetrate its structure. If the crystal was already formed it was not possible to obtain highly concentrated emulsions because the structure of the crystal is very compact and does not allow the disperse phase to penetrate. The following chart shows the experiments account conducted.

Table 1: Experiments performed to characterize the emulsion behaviuor. Studied range was N=9001400rpm; $q=5-10 \mathrm{~mL} / \mathrm{min}$; decane: $80-90 \%$

\begin{tabular}{ccc}
\hline $\mathbf{q}(\mathbf{m L} / \mathbf{m i n})$ & $\mathbf{N}(\mathbf{r p m})$ & Decane $(\mathbf{w t} \%)$ \\
\hline \hline 7,5 & 1575 & 85 \\
10 & 900 & 80 \\
5 & 900 & 80 \\
7,5 & 730 & 85 \\
7,5 & 1150 & 85 \\
5 & 1400 & 80 \\
10 & 1400 & 80 \\
7,5 & 1150 & 93 \\
7,5 & 1150 & 85 \\
10 & 1400 & 90 \\
3,3 & 1150 & 85 \\
5 & 1400 & 90 \\
10 & 900 & 90 \\
7,5 & 1150 & 76 \\
5 & 900 & 90 \\
11,7 & 1150 & 85 \\
7,5 & 1150 & 85 \\
7,5 & 1150 & 85
\end{tabular}

The emulsions presented a white color due to the difference of the refraction index between the external phase and the disperse phase ${ }^{12}$ as well as a high stability, provided by a continuous phase with a very compact structure. The samples were preserved in perfect condition for more than six months, more stables than other systems ${ }^{13}$.

\section{Characterization of the emulsion: drop diameter}

The obtained emulsions have very small drop diameters and the samples had to be diluted in a drop of continuous phase in the microscope slide for the drops to get apart and could be observed by optical microscope. The figure shows a photo of a sample of the experimental design central point. 

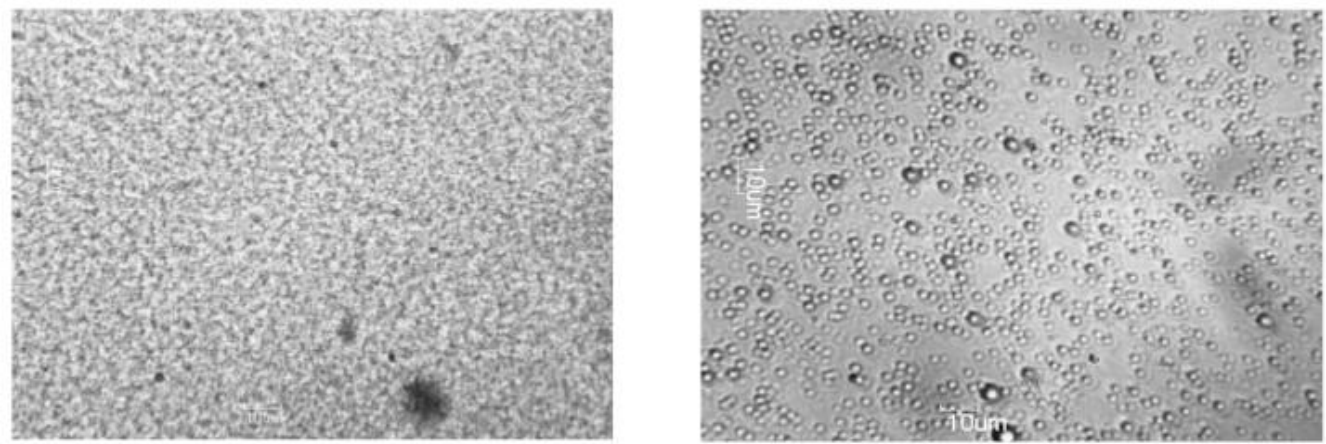

Figure 4: Image of the 80\% decane sample, relation $S: W$ 0,3:0,7 (a) Image of the emulsion not diluted (b) Image of the emulsion in the microscope slide diluted with a drop of water (continuous phase).

To determine the diameter of the drops five photos of each sample were taken to be measured through Motic Plus 2.0. One hundred drops were measured in each photo, so a total of 500 drops per sample were studied. The experimental design was analyzed through the Statgraphics 5.1 software and the result was that only a single significant variable affected to the drop diameter, adding speed of the disperse phase.

\section{Standarized Pareto Chart for d}

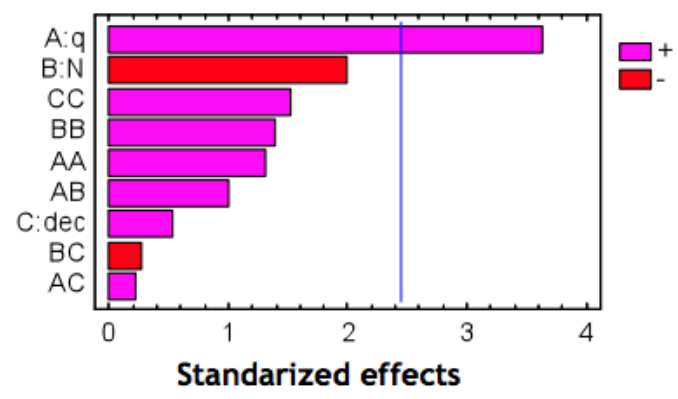

Figure 5: Pareto chart for drople diameter

\section{Emulsion characterization: Rheology}

Oscillatory linear viscoelastic experiments were performed, since they give us information about the behavior of liquid crystal and gel emulsions structure under stress without damaging the internal structure because small deformation was applied. The viscoelastic parameters and the yield stress were evaluated as a function of increasing decane concentration, varying the addition rate and the stirring speed.

It was determined that, as shown in previous study of Kunieda et al. ${ }^{[13],}$ the higher the emulsion dispersed volume is, the lower strain is needed to break it down.

In all the cases it was observed that G' value is higher than the G', and both were practically frequency independent, typical of a gel. It is due because the samples have a solid behaviour instead of a liquid behavior, so external phase was formed by a cubic liquid crystal. 


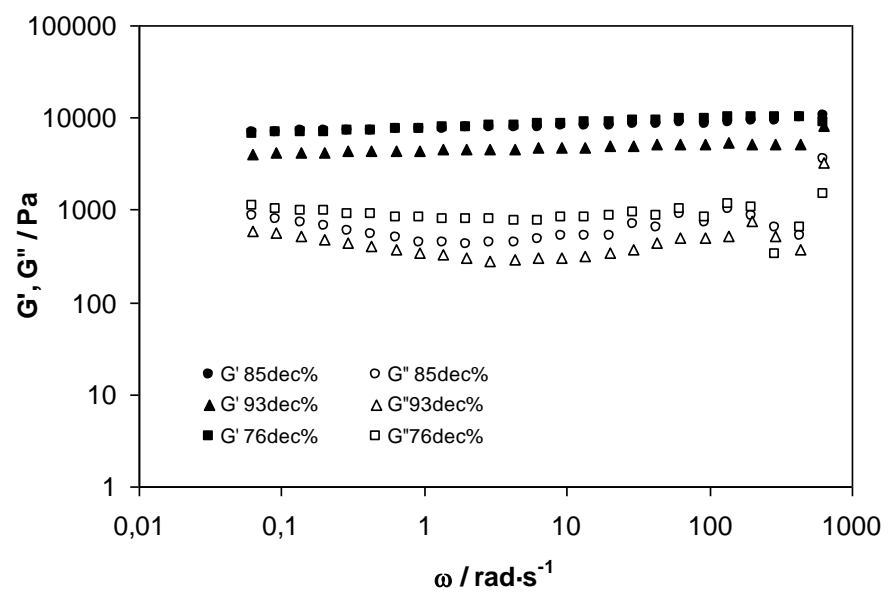

Figure 6: Frequency sweep test data of the elastic ( $G$ ') (filled symbols) and viscous (G") (open symbols) modules or $I_{1}$ phase at $25^{\circ} \mathrm{C}$ at three different weight fractions, as indicated

Pareto chart shows the only variable with a significant importance to $G^{\prime}$ and $G$ " is the dispersed phase volume, as it is shown.

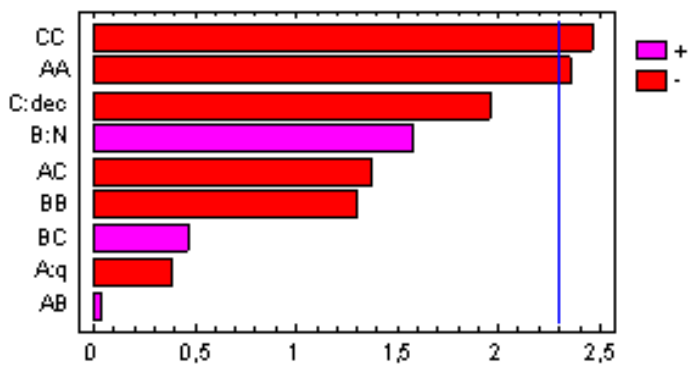

Standarized effects

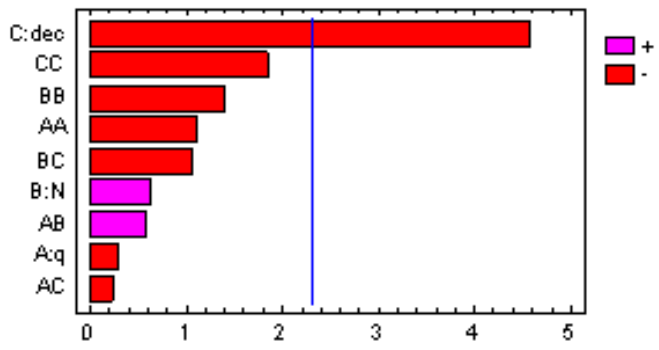

Standarized effects

Figure 7: On the left pareto chart for elastic modulus $\left(G^{\prime}\right)$, on the right pareto chart for viscous modulus (G")

The figure 8 and figure 9 indicate highly concentrated emulsion with a cubic liquid crystal phase has lower G' and G" when dispersed phase increases.

\section{Yield stress}

All the samples were subject to a yield stress test. All of them shared the same behavior as the samples did not flow in any moment until they broke at a stress limit point. As the samples never flowed it is not proper to talk about yield stress, and it seems more appropriate to talk about breaking point. The absence of a yield stress shows that the sample has the typical behavior of a solid. Through statistic 
analysis it was determined that only the disperse phase among the three studied variables had a meaningful influence on the breaking point (Figure 10).

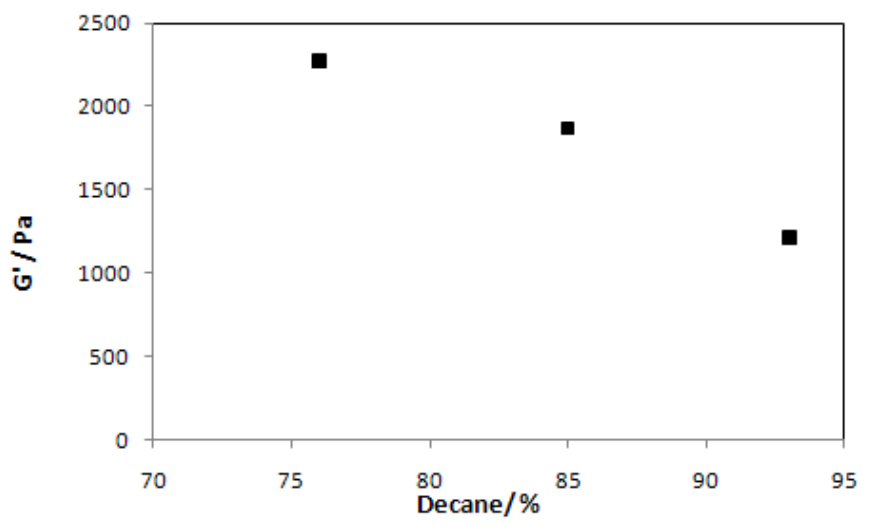

Figure 8: Influence of dispersed phase (decane) in the elastic moduli ( $G$ ').

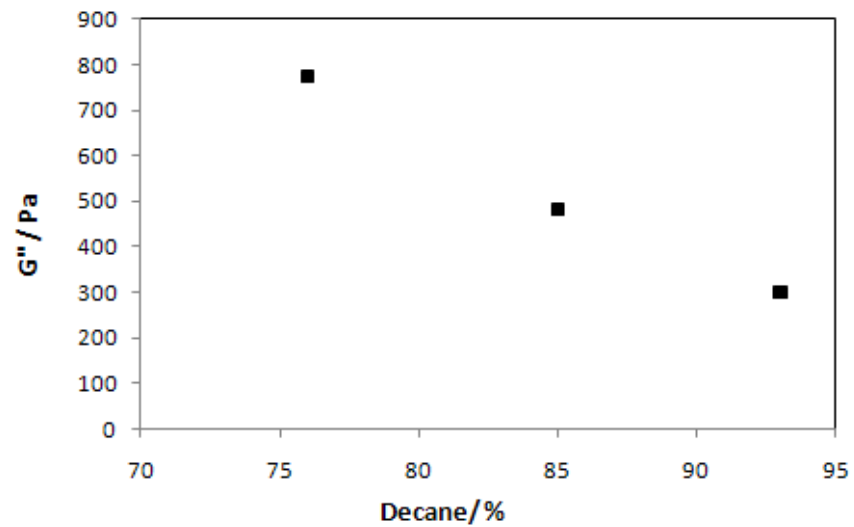

Figure 9: Influence of dispersed phase (decane) in the viscous moduli (G").

\section{Standarized Pareto Chart for rupture point}

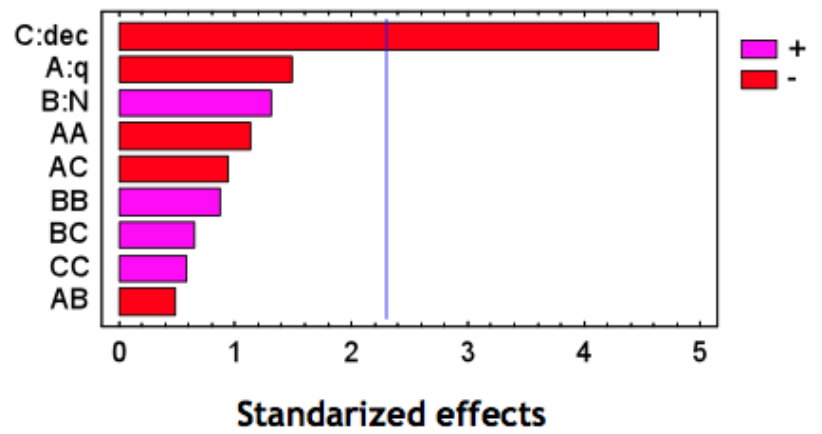

Figure 10: Pareto chart for emulsion rupture point. It shows the significant variable is dispersed phase. 
J. Nepal Chem. Soc., vol. 30, 2012

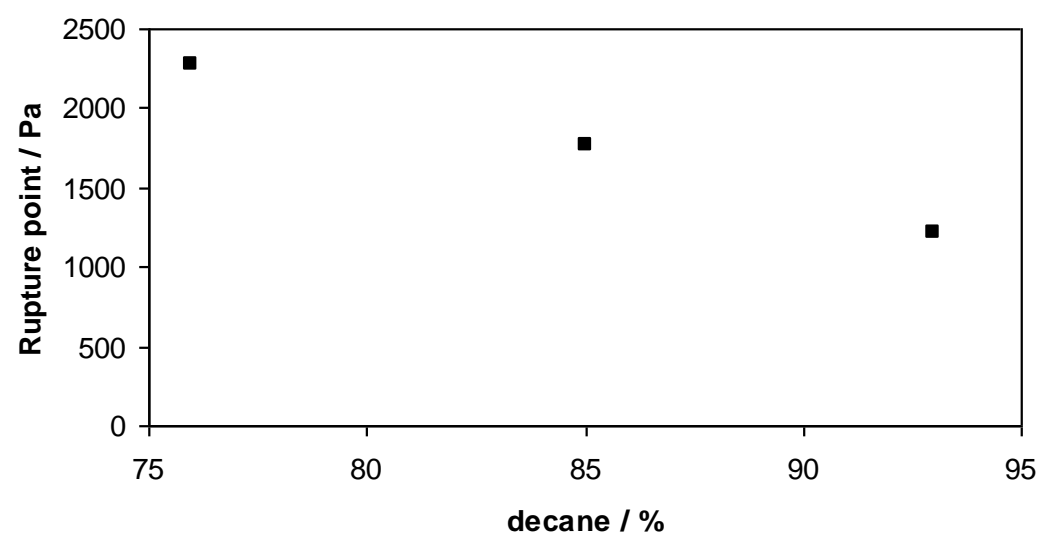

Figure 11: Influence of dispersed phase (decane) in the rupture point.

\section{Caracterization of the meso/macroporous material}

The obtained material was characterized through SEM in order to observe the presence of macropores (Figure 12). Through BET technique the specific area of the material is obtained. In this case it is $520 \mathrm{~m}^{2} / \mathrm{g}$.

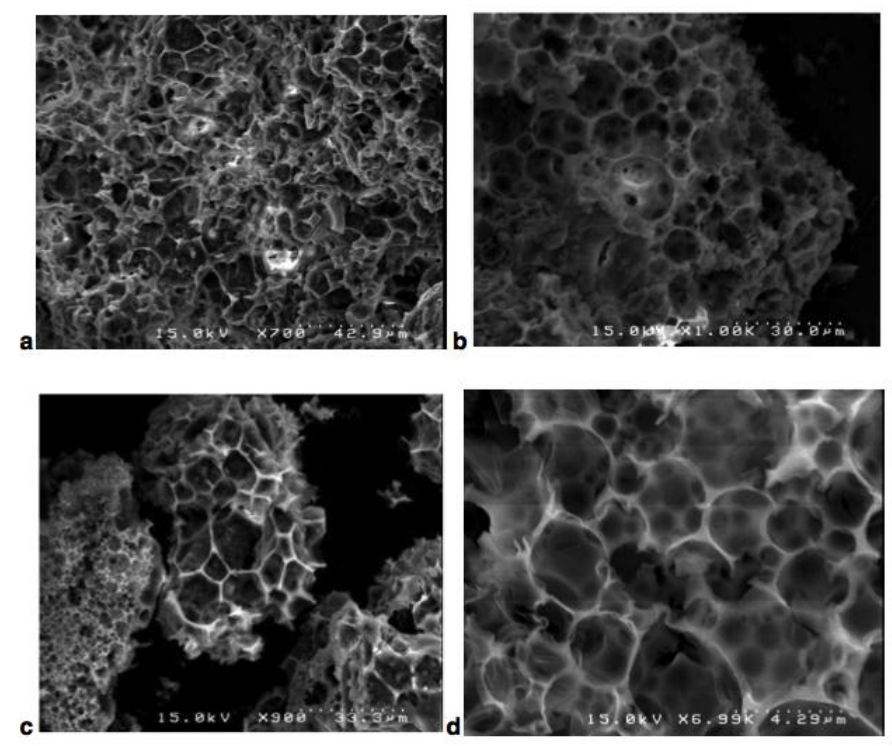

Figure 12: SEM images a different magnifications of silica meso/macroporous material synthesized with TEOS over the emulsion template

\section{Preparation of catalyst}

The meso/macroporous material obtained must be conditioned as an able catalyst support. In the first stages it is important to ensure it has no water washing it with methanol. After a first dry process in the 
oven at atmospheric pressure and at $90^{\circ} \mathrm{C}$ during two hours, it is left overnight a void oven $110^{\circ} \mathrm{C}$ and 10 mbar. Soaking is carried out for depositing the active phase of the catalyst on the support. The meso/macroporous material was soaked with Nafion polymer $\left(\mathrm{C}_{7} \mathrm{H} \mathrm{F}_{13} \mathrm{O}_{5} \mathrm{~S} \mathrm{C}_{2} \mathrm{~F}_{4}\right)$. This polymer is normally used as catalyst (Friedel-Crafts, esterifications, etc). Nafion was soaked with the mesoporous material MCM-41 supplied by Sigma-Aldrich. Nafion was soaked on the meso/macroporous material was double than the amount soaked in the commercial MCM-41.

It is proved that when increasing the channels and cavities of the material the penetration of the molecules is favored, in this case the Nafion, and probably in applications like the controlled liberation of medicines the transference of the material will be increased.

\section{Conclusions}

The present study was focused on the phase behavior (water/C12EO23/decane), emulsion formation and meso/macroporous material formation. The formation of bicontinuous cubic crystal liquid was observed and confirmed by SAXS as well as the formation of highly stable emulsion with a structured bicontinuous liquid crystal in the continuous phase. The formation of the emulsion was so difficult due the preparation and composition variables affected the process and only a narrow range of formation was determined. Rheological tests showed a typical solid behavior where G' and G' decreased when the dispersed phase increases, and G' is always higher than G".

Meso/macroporous material was synthesized from the emulsion, and characterized by SEM and BET. The material presented a high specific area and a porous size in a microscale range. These porous allowed a better soak of the nafion polymer (used as a catalyst). Soak results were much better than the impregnation of a commercial MCM-41.

\section{Acknowledgement}

This study would not have been possible without the financial support from the Spanish Ministry of Science and Innovation (MICINN) within the framework of the project CTQ2008-06892-C03-03/PPQ.

\section{References}

1. V.G Babak; M.J Stebe; J. Dispersion. Sci. Technol., 2002, 23, 1-3, 1-22

2. V. Meynen, P. Cool , E.F. Vansant; Microporous and Mesoporous Materials 125 (2009) 170223

3. Suraj Chandra Sharma a, H. Kunieda, Jordi Esquena, C. Rodríguez Abreu. Journal of Colloid and Interface Science 299 (2006) 297-304

4. C. T, Kresge ; M, E. Leonowicz, ; W J Roth, ; Nature Vol 359 (1992)

5. Zhong-Yong Yuan and Bao-Lian Su; J. Mater. Chem, 2006, 16, 663-667

6. Soo-Hyun Kim, Chang-Kyo Shin, Cheo-Heung Ahn. J Porous Mater (2006) 13: 201-205

7. J. J. Chiu, D. J. Pine, S. T. Bishop and B. F. Chmelka, J. Catal., 2004, 221, 400

8. Galo J. de A. A. Soler-Illia, Clément Sanchez, Bénédicte Lebeau and Joël Patarin; Chem. Rev. 2002, 102, 4093-4138

9. G.E.P. Box, W.G. Hunter, J.S. Hunter Estadística para experimentadores. Ed. Reverté S.A. Barcelona 1989.

10. K, Shigeta; U, Olson; H, Kunieda. Langmuir 2001 17, 4717

11. N, Kanei; H, Watanabe; H. Kunieda. J Oleo Sci.2003, 52, 607

12. Rodriguez-Abreu, García Roman and H, Kunieda. Langmuir 2004, 20, 5235-5240

13. M.M Alam; Y, Sugiyama; K, Watanabe; K, Aramaki; J. Colloid Interface Sci. 2010, 341, 267272 